\title{
Flow and Yield Characteristics of Yield Stress Fluids Using Hysteresis Loop Test Below Slip Yield Point
}

https://doi.org/10.1515/arh-2020-0115

Received Oct 16, 2020; accepted Mar 23, 2021

\begin{abstract}
The flow characteristics of an $\alpha$-gel O/W emulsion, which is a yield stress fluid, was investigated. The hysteresis loop test was conducted for the strain below the slip yield point, and the single relaxation Maxwell model was used to fit the experimental data. Using these methods, the shear-rate dependence, stress dependence, and time dependence of the viscoelastic properties of the sample were evaluated in the region below the slip yield point. The shear-rate dependence induced by the stress-ramp rate and the stress dependence from the maximum applied stress influence the viscoelastic characteristics below the slip yield point in terms of the flow history. However, the time dependence of the viscoelastic characteristics could not be confirmed for any creep time. The yield stress measured in the stress-ramp test increases with the stress-ramp rate owing to the contribution of the viscous strain from the flow history.
\end{abstract}

Keywords: Yield stress fluids, Hysteresis loop test, Maxwell model, $\alpha$-gels

\section{Introduction}

Yield stress fluids are a kind of fluid defined by Bingham in 1922 [1]. However, research on such fluids has not been very popular until recently. From the early 2000s, research on yield stress fluids started to increase because products exhibiting yield stress have been developed in various industries. Moreover, the measurement accuracy of experimental devices has improved allowing low stresses and strains to be measured [2]. The structure of yield stress flu-

\footnotetext{
*Corresponding Author: Tsutomu Takahashi: Department of Mechanical Engineering, Nagaoka University of Technology, 1603-1 Kamitomioka, Nagaoka, Niigata 940-2188, Japan; Email: ttaka@nagaokaut.ac.jp

Yasunori Sato, Yukinobu Sugihara: Department of Mechanical Engineering, Nagaoka University of Technology, 1603-1 Kamitomioka, Nagaoka, Niigata 940-2188, Japan
}

つ Open Access. (C) 2021 Y. Sato et al., published by De Gruyter. (Cc) BY License ids can be explained by the bond force between dispersed particles [3] and the gel-network [4]. Note that such fluids do not flow until the stress applied exceeds a certain value (i.e., the yield stress). The functionality of various products encountered in our daily life, such as ink [5], food products [6], medicines [7], and cosmetics [8], is enhanced by the application of yield stress. For instance, hand creams are easier to handle if their macrostructure is retained while taking the cream out of the bottle [9]. However, during application on the skin, the cream can be easily extended by supplying a low yield stress. Additionally, the yield stress affects storage stability in the long term by trapping bubbles and liquid droplets in the surrounding fluid [10]. Recently, products exhibiting yield stress have been in widespread use, even in the currently established industries such as bio-printing [11], rocket fuel manufacturing [12], 3D printing [13], and hydrogel manufacturing for drug delivery systems [14].

To measure the yield behavior of soft matter, the following tests have been proposed: the steady viscoelastic test, which measures the steady value with which the shear-rate or the shear stress increases (or decreases); the creep stress test, which applies a constant shear stress for a long time; the stress sweep test, which applies a sinusoidal stress; and the stress-ramp test, which causes a transient flow [15]. In general, yield stress fluids can be classified into two types using the steady viscoelastic test. The first category is simple yield stress fluids such as Carbopol-gels [16]. When the steady viscoelastic test is performed on such fluids, the results under which the shear-rate increases or decreases are completely consistent. Therefore, the viscosity depends only on the shear-rate and the yield stress is an inherent property of the material [17]. The second category is thixotropic yield stress fluids, which are mainly observed as various foams and gels. When the steady viscoelastic test is performed on these fluids, the results under which the shear-rate increases or decreases are not consistent. Thus, the viscosity is not only a function of the shear-rate but also of the accumulated strain, applied stress, and static time. This effect is called the flow history. The flow history affects the rheological behavior of thixotropic fluids because the 
macro network structure is destroyed over time by the flow in such fluids.

According to a study on the relationship between static time and yield stress characteristics, Coussot [18] revealed that the yield stress increases with static time by conducting a viscosity bifurcation test on a bentonite suspension in water. Ovarlez [19] reported that the yield stress increases with the applied stress after the application of a pre-shear on a carbon fiber suspension. Gerenald [20] reported the presence of two timescales that depend on the flow time and presented the findings of how the viscosity and yield stress changed during the creep test performed on a colloidal gel mixed with carbon particles. Based on these results, Larson [21] pointed out that a change in viscosity plays a major role in changing the yield stress. Note that the flow characteristics below the yield point are mainly measured using the small amplitude oscillatory shear technique, which applies a small sinusoidal stress or strain; however, it has been reported that the yield behavior itself is influenced by the flow time, pre-shear, and static time as above.

The yield stress in fluids can be classified as slip and true. Slip yield stress is the yield stress induced by any of the flow inhomogeneities at the wall, as the name suggests. True yield stress is the yield stress of the original sample that is related to the interparticle bond force of the underlying microstructure [22]. In the context of slip yield stress, Bingham [1] had already pointed out the existence of a lubricant layer that forms in the suspension. It is important to understand the slip behavior in yield stress fluids to reduce the resistance in tube flows, as required in 3D-printing, and to increase the flow stability on smooth surfaces. Note that slip yield stress is exhibited by condensed suspensions, softjammed systems, and emulsions [23]. Meeker et al. [24, 25] reported the formation of a lubricant layer close to the wall surface of their soft-jammed sample and found that the relationship between shear stress and slip velocity can be represented by a quadratic function. Ballesta et al. [26, 27] carried out comprehensive rheological measurements on hard-sphere colloidal suspensions and reported that the slip behavior is suppressed when the wall roughness (of the scale of the dispersed particle size) is high and the slip is maintained over the yield stress [26, 27]. Following these works, Zhang et al. [28], in a crucial study, clarified the physical mechanism behind the wall slip using rheological measurements and MRI velocimetry. They showed that the slip yield stress is induced by the edge effect related to the evaporation at the wall surface and the pinning or adhesion of the dispersed particles to the wall.

In a study that focused on determining the conditions for preventing slip behavior in the solid regime of yield stress fluids, Coussot et al. [29] reported the results of their creep stress experiments with different stress levels on the aging as well as the behavior of the fluids in the solid and liquid regimes. The creep tests in this study distinguished two regimes, namely, the solid regime, where the strain approached a constant value and the liquid regime, where the strain continued to increase. This study also showed that, even in the solid regime, the sample behaved as a viscoelastic solid because plastic deformation set in over critical stress.Based on this study, Tabuteau et al. [30] showed that the flow model of yield stress fluids are in good agreement with the results of the spin coating experiments. Among the theoretical studies, Saramito et al. [31] constructed a flow model combining the Herschel-Bulkley and Oldroyd models, which can predict the yield behavior well compared to previous models. To understand the LAOStress behavior, even in the solid regime of the yield stress, Dimitriou et al. [32] proposed a new model that can explain the strain stiffening just before yielding by introducing plasticity and elasticity into the Herschel-Bulkley model. N'Gouamba et al. [33] explored the structural state of soft-jammed materials using creep tests and the corresponding recovery tests. They found that the material exhibited a constant elasticity up to the true yield stress in the solid regime, based on this they proposed a generic model of solid-state dynamics with elasto-plasticity.

Considering the above background, in this study, we aim to clarify the viscoelastic characteristics in the solid regime under conditions in which the slip yield behavior is exhibited rather than true yield behavior, unlike in previous studies. The slip yield stress is induced by the interaction between the solid surface and the material. To conduct this experiment, a smooth and a rough surface were used for result comparison. Therefore, a stainless plate and a sandpaper (J1000) attached plate were selected. The experimental procedure followed and the analysis performed to evaluate the viscoelastic characteristics of the transient flow and its effect on the yield stress are proposed. Specifically, the hysteresis loop test is conducted for a range of stresses below the slip yield point using parameters such as the maximum applied stress, stress ramp rate, and creep time. Considering that the viscous strain is much more resistant compared to the plastic strain on the smooth plate where the slip occurs, the strain behavior is fitted using the single Maxwell relaxation model. The stress dependence, shear-rate dependence, and time dependence of the viscoelastic characteristics below the slip yield point are also investigated. The impact of these characteristics on the slip yield stress is clarified in terms of the viscosity and elasticity. Finally, these results are compared with those corresponding to true yield stress, and the effectiveness of 
our experimental procedure and analysis in understanding the yield behavior is discussed.

\section{Experiments}

\subsection{Rheometer}

We used the stress-controlled twin-drive rheometer MCR702 (Anton Paar Co. Ltd.) to conduct the rheological tests mentioned in this section. In this rheometer, the driving motor and transducer are located at the top and bottom, respectively, such that it can control the top and bottom plates freely. We conduct our experiments in the "2EC separate transducer mode," which allows the rotation of the bottom plate while the top plate remains fixed. The rheometer consists of two parallel stainless steel disks with a plate diameter of $25 \mathrm{~mm}$ each. The gap between the top and bottom plates was set at $0.5 \mathrm{~mm}$. A stainless steel plate of Ra 0.8 was used as a smooth surface, while a stainless steel plate of Ra 16 obtained by gluing the sand paper (J1000) was used as a rough surface in order to prevent the slip behavior.

\subsection{Stress-ramp and stress sweep tests}

In this study, the yield behavior is measured using the stress-ramp and stress sweep tests. In the stress-ramp test, the shear stress increases at a constant stress-ramp rate from zero to a certain value (i.e., the maximum applied stress). For yield stress fluids, the stress-ramp test is useful for evaluating the critical stress and strain, that is, the yield stress and yield strain at which the sample deforms significantly. The stress-ramp rate parameter was set to $0.1,0.2$, $0.5,2.0,5.0$, and $10.0 \mathrm{~Pa} / \mathrm{s}$, and the maximum applied stress was $100 \mathrm{~Pa}$. A stress sweep test, in which a small sinusoidal stress is applied, was also conducted.

A stress-ramp rate of $0.5 \mathrm{~Pa} / \mathrm{s}$ was applied before the actual stress-ramp test as a pre-shear to eliminate the influence of loading the sample. After the application of the pre-shear, the static time, which is the time required by the sample to flow, was sufficient for the sample to recover and exhibit the yield behavior again. The maximum applied stress during the pre-shear was $100 \mathrm{~Pa}$ to prevent the sample from scattering under the high rotation. The experimental temperature was maintained at $25^{\circ} \mathrm{C}$.

\subsection{Sample}

An $\alpha$-gel O/W emulsion with an $\alpha$-gel network formed between the emulsified particles using ion-exchange water as the dispersant and liquid paraffin as the dispersing medium was used in this study [34]. An $\alpha$-Gel is an aggregate formed by the interaction of a higher alcohol and a surfactant, which has a structure similar to that of lamellar liquid crystals. $\alpha$-Gels are used to coordinate the flow characteristics of various products in the field of cosmetics [35]. In this study, an $\alpha$-gel was prepared in a three component system consisting of behenyl alcohol, sodium stearoylmethyl taurine, and water. The water in the sample was kept stable in the lamellar phase, which is possible at a maximum water concentration of $85 \%$ [34]. To confirm the generality of the yield stress behavior, a colloidal gel with dispersed clay particles, which is different from the $\alpha$-gel $\mathrm{O} / \mathrm{W}$ emulsion, was used. We also compared our results by using silicone oil KF-96-3000 cSt (Shin-Etsu Chemical Co., Ltd.) as the sample. Although the rheological measurements were conducted for more than $2 \mathrm{~h}$, the sample was not dry. Note that our pre-experiments showed that there was no influence of drying the sample in this study [36].

\subsection{Outline of hysteresis loop test below yield point and single relaxation Maxwell model}

The hysteresis loop test is a measurement method in which the shear stress increases from zero to a certain value and then decreases back to zero again. This test can evaluate the time dependence of the thixotropic and viscoelastic characteristics of the sample, both before and after its destruction [37]. Figure 1 depicts an outline of the hysteresis loop test, which is fundamental to the present study. Note that for the hysteresis loop in this study, the strain is measured by increasing or decreasing the stress at a ramp rate below the yield stress.

According to the simple model for a yield stress fluid, it behaves as a rigid body or an elastic solid in the low stress region below the yield point. However, in reality, it exhibits a viscous behavior in this regime such that the generated strain is included in both the elasticity and viscosity; whereas, the permanent strain is included in the viscosity. Thus, the single relaxation Maxwell model was used for fitting, as it includes both the elasticity $E$ and viscosity $\eta$ as parameters. Note that the contribution of the strain for each fluid was considered separately. The single relaxation Maxwell model in a simple shear flow is given 


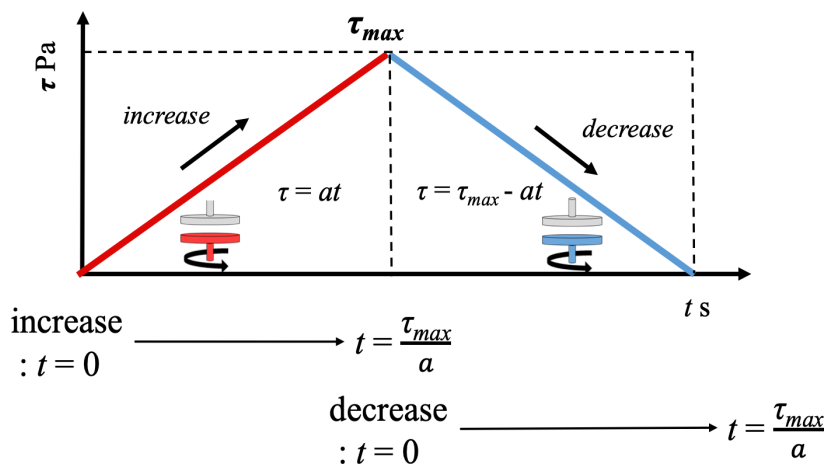

Figure 1: Outline of the hysteresis loop test. During the increasing segment, the shear stress increases from zero to the maximum applied stress at a constant rate. During the decreasing segment, the shear stress decreases from the maximum applied stress to zero at the same constant rate

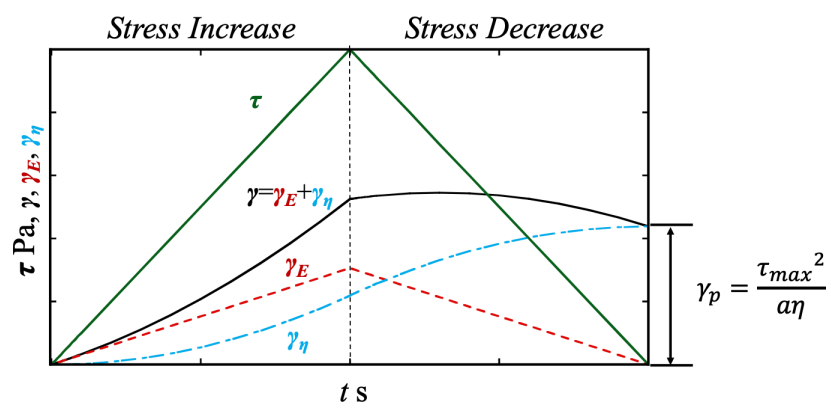

Figure 2: Strain response in the hysteresis loop test. The elastic strain is perfectly recovered, and the viscous strain is retained as the permanent strain $\gamma_{p}$ at the end of the hysteresis loop test

by the following equation:

$$
\tau+\lambda \frac{d \tau}{d t}=\eta \frac{d \gamma}{d t}
$$

where the relaxation time $\lambda$ is the ratio of the elasticity $E$ and viscosity $\eta$ such that

$$
\lambda=\frac{\eta}{E}
$$

The shear stress during the increasing segment of the hysteresis loop is given by $\tau=a t$. Here, $a$ is the stress-ramp rate. The strain $\gamma_{u p}$ in the increasing segment can be obtained by using equation (2) and $\tau=a t$, such that

$$
\gamma_{u p}=\frac{\alpha}{\eta} \int\left(t+\frac{\eta}{E}\right) d t=\frac{\alpha}{\eta}\left(\frac{1}{2} t^{2}+\frac{\eta}{E} t\right)+C t
$$

where, $\gamma_{u p}=0$ at $t=0$, and the constant of integration $C$ is also zero. Thus, we can write

$$
\gamma_{u p}=\frac{a}{\eta}\left(\frac{1}{2} t^{2}+\frac{\eta}{E} t\right)
$$

Note that $E$ and $\eta$ are calculated by fitting the experimental data using the least squares method. Next, the strain $\gamma_{\text {down }}$ in the decreasing segment of the hysteresis loop was obtained. Noting that the shear stress is $\tau=\tau_{\max }-a t$ in the decreasing segment, we can write

$$
\begin{aligned}
\gamma_{\text {down }} & =\frac{1}{\eta} \int\left\{\tau_{\max }-\alpha(t+\lambda)\right\} d t \\
& =\frac{1}{\eta}\left\{\frac{a}{2} t^{2}+t\left(\tau_{\max }-a \lambda\right)\right\}+C
\end{aligned}
$$

where, if time at the start of the decreasing segment is $t=0$, then the strain in this segment is $\gamma_{\text {down }}=\frac{\tau_{\max }{ }^{2}}{2 a \eta}+\lambda \frac{\tau_{\max }}{\eta}$. Thus, the constant of integration becomes $C=\frac{\tau_{\max }{ }^{2}}{2 a \eta}+\lambda \frac{\tau_{\max }}{\eta}$.

Therefore, equation (5) can be written as

$$
\gamma_{\text {down }}=\frac{1}{\eta}\left\{\frac{1}{2} \alpha t^{2}+t\left(\tau_{\text {max }}-\alpha \frac{\eta}{E}\right)\right\}+\frac{\tau^{2} \text { max }}{2 \alpha \eta}+\frac{\eta \tau_{\text {max }}}{E^{2}}
$$

The elasticity and viscosity in the segment of decreasing stress are calculated using the least squares method in the same manner as for the increasing segment. Note that because the rheological characteristics are different for the segments of increasing and decreasing stress in the hysteresis loop, we denote the coefficients in the increasing segment as $E_{u p}$ and $\eta_{u p}$ and those in the decreasing segment as $E_{\text {down }}$ and $\eta_{\text {down }}$ to distinguish between them. The permanent strain $\gamma_{p}$ that is retained at the end of the test is obtained by setting $t=\tau_{\max } / a$ (which is the time at the end of the test) in equations (4) and (6) as follows

$$
\gamma_{p}=\gamma_{\text {up }}+\gamma_{\text {down }}=\frac{\tau^{2} \text { max }}{\alpha \eta}
$$

Figure 2 depicts an example of the strain response to the hysteresis loop test according to the single relaxation Maxwell model. First, for the elastic component as per Hooke's law, the obtained strain shows the same trend in both the increasing and decreasing segments. Next, for the viscous component, the strain increases in both the increasing and decreasing segments. Note that it is the strain in the viscous component that is retained as the permanent strain at the end of the test. The solid black line in the figure represents the total strain, which combines the strain responses of the elasticity and viscosity in the single relaxation Maxwell model. Thus, although the elastic strain is zero when the stress $\tau=0 \mathrm{~Pa}$, the viscous strain is not, and hence, the latter appears as the permanent strain in the single relaxation Maxwell model. The strain is stored predominantly as elastic energy in the hysteresis loop for yield stress fluids because the elastic component is dominant below the yield point, as observed from Figure 2; thus, the elastic strain can be completely recovered in accordance to Hooke's law in the segment of decreasing stress. Meanwhile, the permanent strain is generated by the viscous component of the strain. 


\section{Results and discussions}

\subsection{Validation of hysteresis loop test using permanent strain}

To set the maximum stress that can be applied during the hysteresis loop test, initially, the true yield and slip yield stresses need to be determined from the results of the stressramp test. Figure 3 illustrates the stress-strain curve and the flow curve obtained for a stress-ramp rate of $0.5 \mathrm{~Pa} / \mathrm{s}$. In the stress-strain curve, the slip yield stress $\boldsymbol{\tau}_{y s}$ and true yield stress $\boldsymbol{\tau}_{\boldsymbol{y} t}$ are approximately $4 \mathrm{~Pa}$ and $20 \mathrm{~Pa}$, respectively. The slip yield stress measured by several creep tests is about $0.2 \mathrm{~Pa}$. The slip yield stress in the stress-ramp test depends on the procedure of increasing the applied stress We ob-

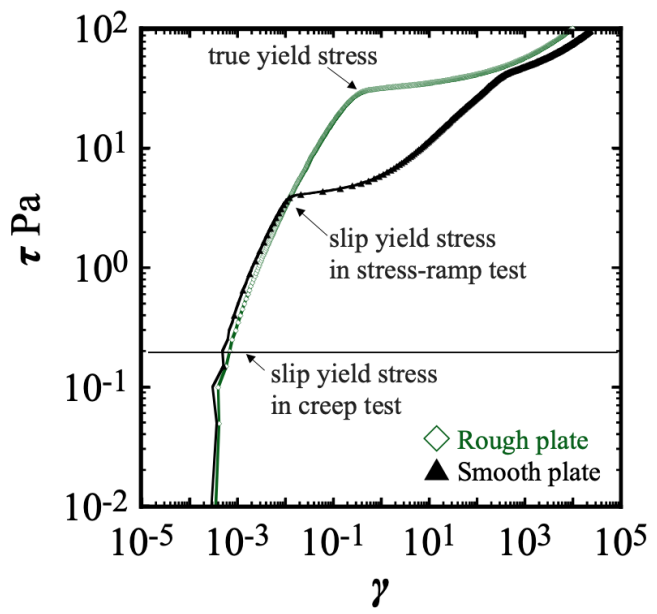

(a) Stress-strain curve

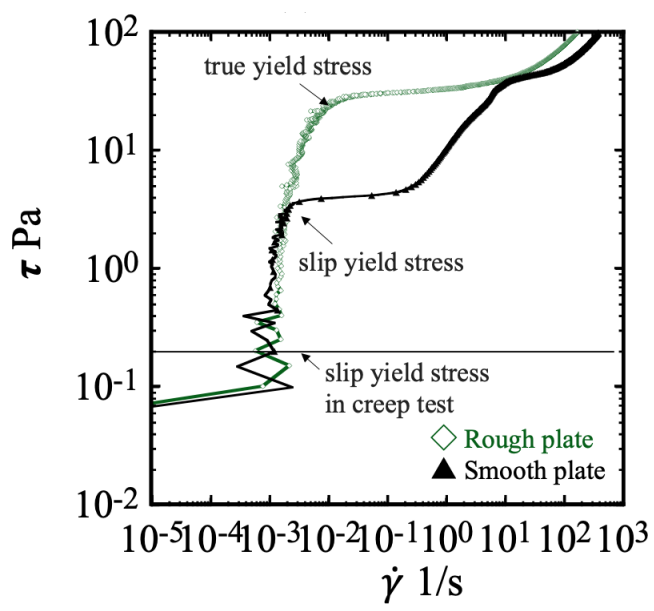

(b) Flow-curve

Figure 3: (a) Stress-strain curve and (b) flow-curve, were obtained from a stress-ramp test with a ramp rate of $0.5 \mathrm{~Pa} / \mathrm{s}$ for plates with different surface roughness. Below the slip yield stress, the slopes for the rough and smooth plates are coherent serve that the slope of the stress-strain curve is constant below both these yield stresses. The elastic characteristic of the fluid is dominant in this region, as discussed in the previous section. The behavior of the stress-strain curve till the slip yield stress is almost the same for the rough (Sandpaper attached) and smooth (stainless steel) plates. From this result, the maximum applied stress for the hysteresis loop test below the slip yield point was determined. To confirm the non-Newtonian behavior of the viscosity below the slip yield stress, the permanent strain was also measured. The permanent strain (at the end of the test) is

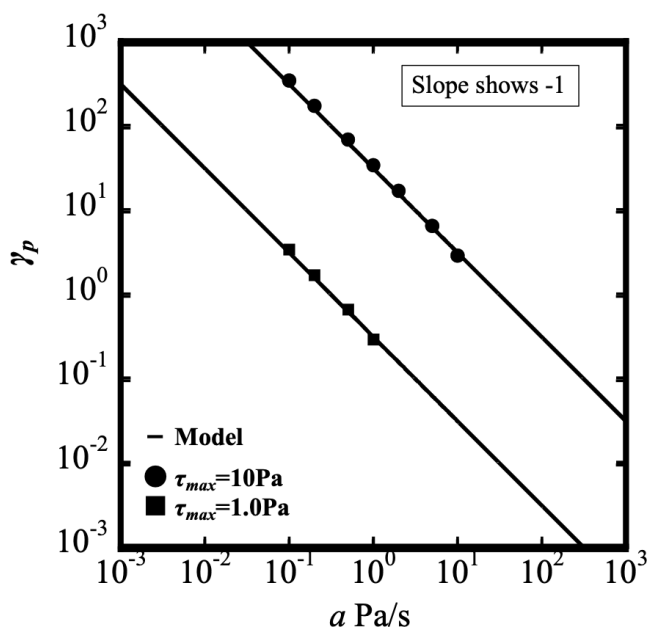

Figure 4: Permanent strain of silicone oil as a function of the stressramp rate in the logarithmic scale. The slope of this function is -1 , which is independent of the external conditions as the sample is a Newtonian fluid

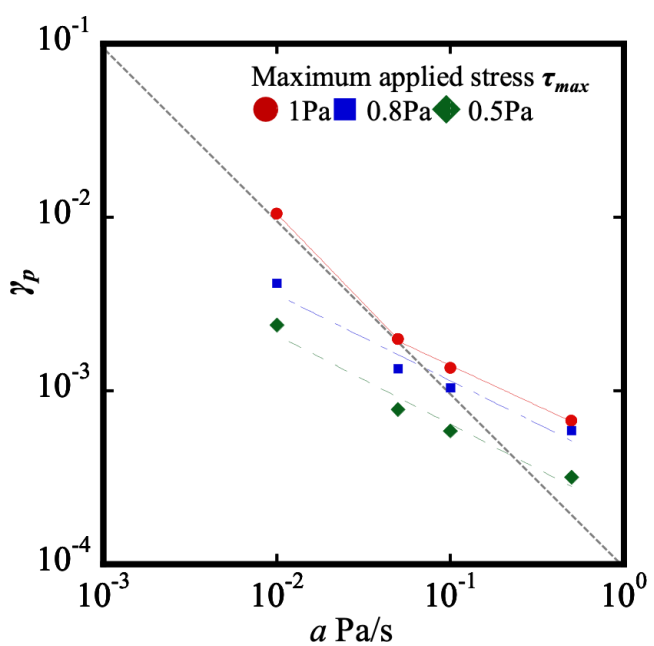

Figure 5: Permanent strain of $\alpha$-gel $0 / \mathrm{W}$ emulsion as a function of the stress-ramp rate in the logarithmic scale. None of the slopes are -1 , as the sample is a non-Newtonian fluid. The slope depends on the maximum applied stress $\tau_{\max }$ (in $\mathrm{Pa}$ ) and the stress-ramp rate $a$ (in $\mathrm{Pa} / \mathrm{s}$ ), as illustrated in Table 1 
Table 1: Slope of the permanent strain for $\alpha$-gel O/W emulsion. The slope depends on the stress-ramp rate and the maximum applied stress. The maximum applied stress $0.8 \mathrm{~Pa}$ and $0.5 \mathrm{~Pa}$ does not show the slope of -1 , unlike Newtonian fluids. The slope in $1.0 \mathrm{~Pa}$ can be divided in two regions $(0.001-0.005 \mathrm{~Pa} / \mathrm{s}, 0.005-0.5 \mathrm{~Pa} / \mathrm{s})$. The former shows the slope of -1 like Newtonian fluids

\begin{tabular}{cc}
\hline $\begin{array}{c}\text { Maximum applied stress } \\
\mathrm{Pa}\end{array}$ & $\begin{array}{c}\text { Slope of permanent strain } \\
\gamma_{\boldsymbol{p}}\end{array}$ \\
\hline 1 & -1.0345 \\
& $(0.001-0.005 \mathrm{~Pa} / \mathrm{s})$ \\
& -0.4639 \\
0.8 & $(0.005-0.5 \mathrm{~Pa} / \mathrm{s})$ \\
0.5 & -0.4955 \\
& -0.5134 \\
\hline
\end{tabular}

required along with the maximum applied stress and stress ramp rate as parameters for the hysteresis loop test. Note that the same experiments were also performed with the silicone oil for comparison with the results obtained for the $\alpha$-gel O/W emulsion.

Figure 4 illustrates the permanent strain of silicone oil as a function of the stress-ramp rate in the logarithmic scale for maximum applied stresses of 1.0 and $10 \mathrm{~Pa}$. According to equation (7), the slope of this function is -1 in the logarithmic scale if the sample is a Newtonian fluid. From Figure 4, we observe that the experimental data for silicone oil lies exactly on the straight line with a slope of -1 even for different values of the maximum applied stress $\tau_{\max }$. Thus, our experiment is validated by the silicone oil data. Next, Figure 5 depicts the permanent strain of the $\alpha$-gel O/W emulsion as a function of the stress-ramp rate in the logarithmic scale for maximum applied stresses of 0.5 , 0.8 , and 1.0 Pa. We observe that the slope in this case except for $1 \mathrm{~Pa}$ is smaller than -1 , as also illustrated in Table 1 . The slope at $1 \mathrm{~Pa}$ can be divided in 2 regimes by reasons discussed below. This implies that the viscosity depends on the stress-ramp rate in the regime below the yield point. Thus, the sample behaves as a non-Newtonian fluid because the viscosity is dependent on the shear-rate. We also observe that as the maximum applied stress increases, the slope approaches -1 ; in particular, at a maximum applied stress of $1 \mathrm{~Pa}$, which is close to the slip yield point, the sample behaves like a Newtonian fluid (slope:-1.0345) in the stressramp rate range of $0.001-0.005 \mathrm{~Pa} / \mathrm{s}$. These results show that the sample enters a state of dominant viscosity as the stress approaches the yield point (although not crossing it), which indicates that the microstructure contributing to the elasticity of the sample is destroyed. Rubens et al. [38] conducted a stress sweep at a constant frequency of a commercial hair gel, which is a thixotropic yield stress fluid. Their Fourier analysis showed that the third-order harmonic component of the strain, that is, the nonlinear response, is large even for an applied stress smaller than the crossover point, where the loss modulus $G^{\prime \prime}$ becomes larger than the storage modulus $G^{\prime}$ and the sample starts to yield. In other words, it is predicted that the strain response of the viscoelastic properties of the sample may change from linear to nonlinear, even when the applied stress is well below the yield stress.

\subsection{Experimental sequences in a stress range below the yield point}

Figure 6 shows three experimental sequences of the hysteresis loop test in a stress range below the yield point in this study. The first parameter varied was the stress-ramp rate,

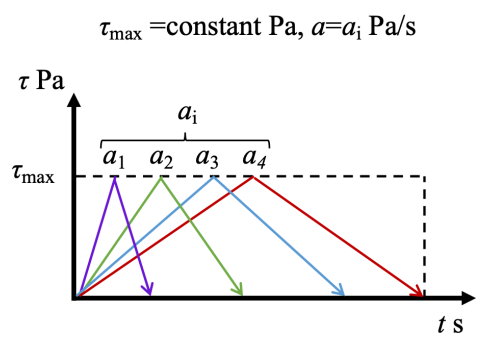

(a) Stress-ramp dependence

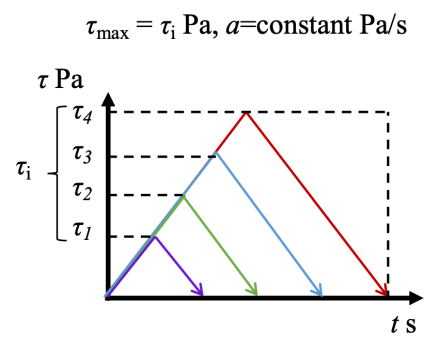

(b) Stress dependence

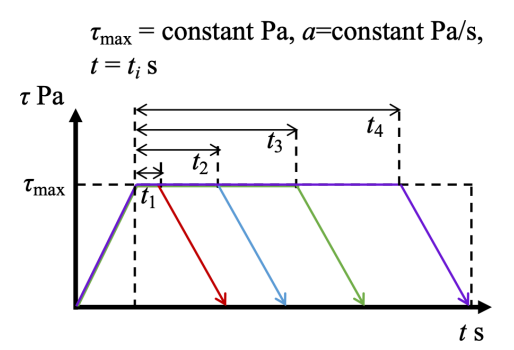

(c) Time dependence

Figure 6: Three sequences for evaluating various dependences of the viscoelastic properties of the sample. (a) The stress-ramp rate dependence, which can further probe the shear-rate and time dependence. (b) The stress dependence, which was studied by increasing the maximum applied stress. (c) The time dependence, which was investigated by increasing the creep time 
as shown in Figure 6(a). We observe that the shear stress increases as well as decreases at a constant stress-ramp rate $a_{i}$ in Figure 6(a). In this case, the time dependence and shear-rate dependence of the viscoelastic characteristics of the sample were investigated by changing the total time of the applied stress and the shear-rate. Next, we varied the maximum applied stress to investigate the change in the viscoelastic characteristics as the applied stress approaches the yield point, as shown in Figure 6(b). Note that the stress-ramp rates in Figure 6(a) are 0.01, 0.05, 0.1, and $0.5 \mathrm{~Pa} / \mathrm{s}$, and the maximum applied stress in Figure 6(b) are 0.5, 0.8, and 1.0 Pa. Figure 6(c) shows a sequence of the creep stress test inserted into the hysteresis loop test. The total time for the creep test $t_{i}$ is the parameter that was varied to investigate the time dependence of the viscoelastic characteristics below the yield point. In Figure 6(c), the stress-ramp rate is $0.1 \mathrm{~Pa} / \mathrm{s}$, the maximum applied stress is $1 \mathrm{~Pa}$, and the creep times are 1.0, 10, 60, 100, and $1200 \mathrm{~s}$.

\subsection{Experimental data of elastic and viscous strains below yield point}

Equations (4) and (6) of the single relaxation Maxwell model were used to fit the strain in the segments of increasing and decreasing stress of the hysteresis loop test. Subsequently, the components of elasticity and viscosity in the measured strain were estimated. Figure 7 shows the elastic and viscous strains in the $\alpha$-gel $\mathrm{O} / \mathrm{W}$ emulsion at a maximum applied stress of $1 \mathrm{~Pa}$ and for stress-ramp rates $0.01,0.05,0.1$ and $0.5 \mathrm{~Pa} / \mathrm{s}$. For the stress increasing segment shown in the left panels of Figures 7(a)-(d), the experimentally obtained strain is in good agreement with that of the single relaxation Maxwell model. Note that because the elastic strain increases linearly and the viscous strain increases quadratically with time, the elastic strain dominates at the start of the test, while the viscous flow is dominant later in time. The right panels of Figures 7(a)-(d) show

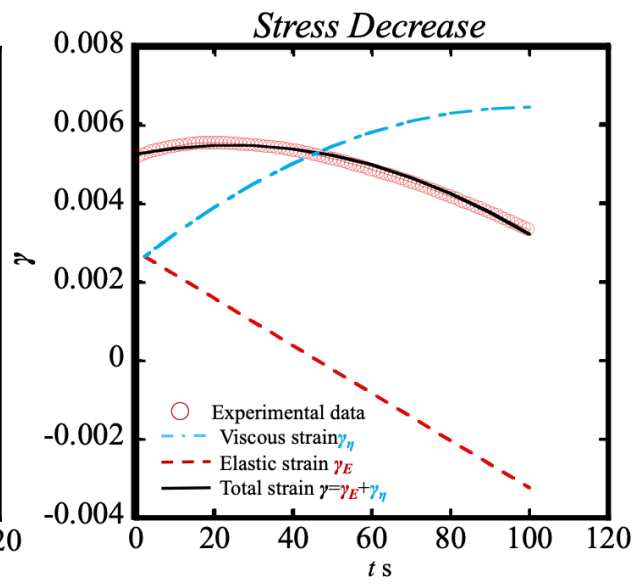

(a) $a=0.01 \mathrm{~Pa} / \mathrm{s}$
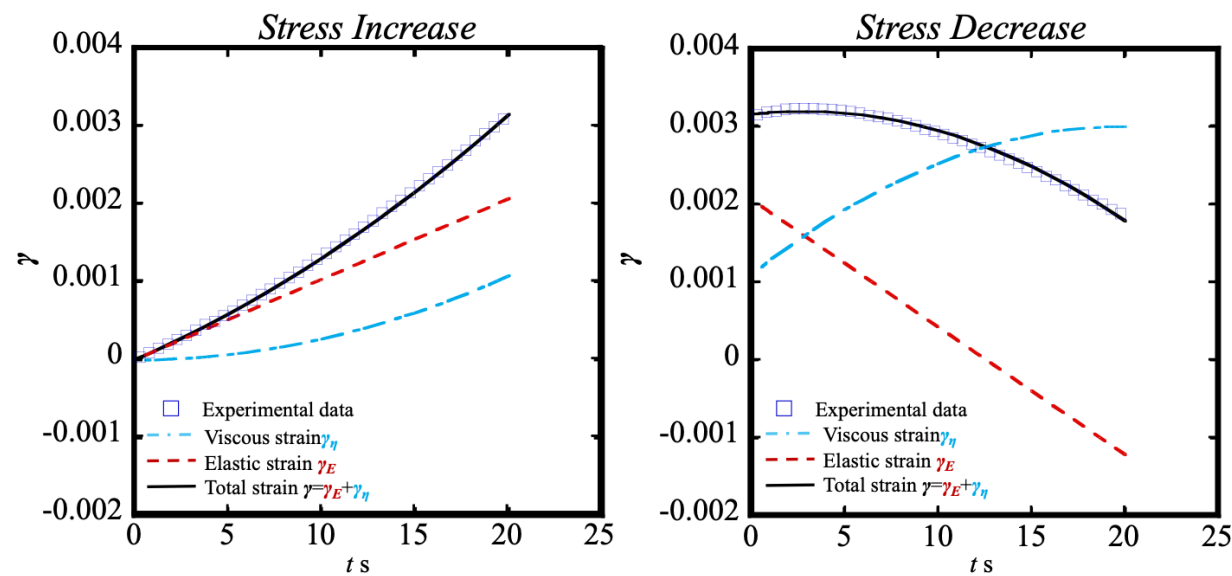

(b) $a=0.05 \mathrm{~Pa} / \mathrm{s}$

Figure 7: Comparison of the elastic and viscous strains obtained from experiments and Maxwell model fitting of the $\alpha$-gel $0 / \mathrm{W}$ emulsion. Stress-ramp rates: (a) $a=0.01 \mathrm{~Pa} / \mathrm{s}$, (b) $a=0.05 \mathrm{~Pa} / \mathrm{s}$, (c) $a=0.1 \mathrm{~Pa} / \mathrm{s}$, and (d) $a=0.5 \mathrm{~Pa} / \mathrm{s}$. The viscoelastic characteristics in the stress decreasing segment is influenced by the flow history in the stress increasing segment 

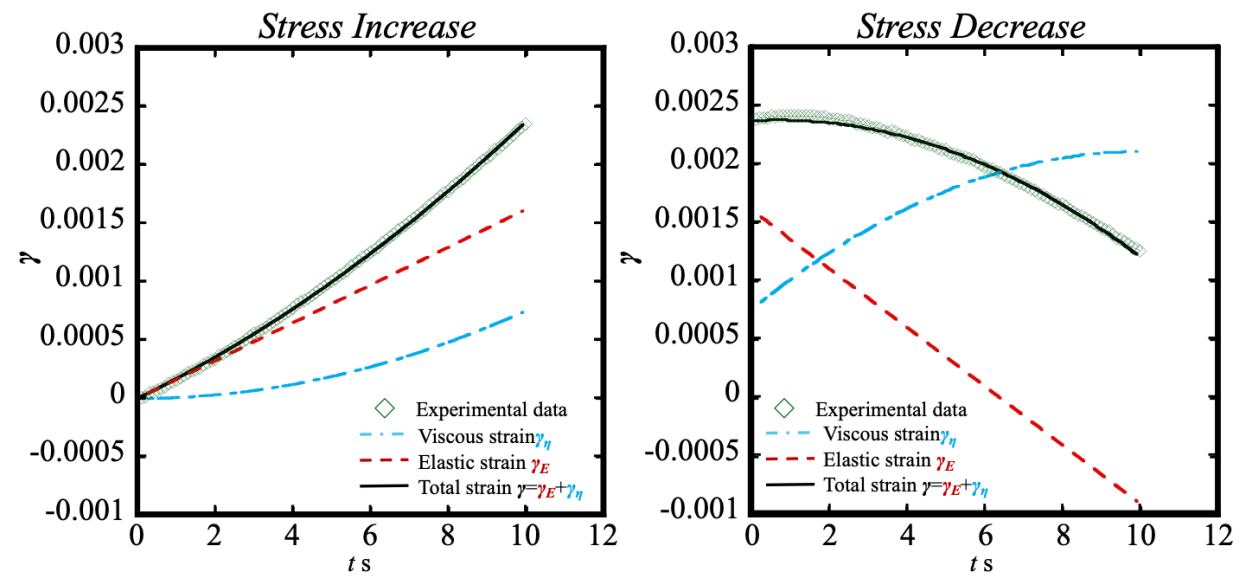

(c) $a=0.1 \mathrm{~Pa} / \mathrm{s}$
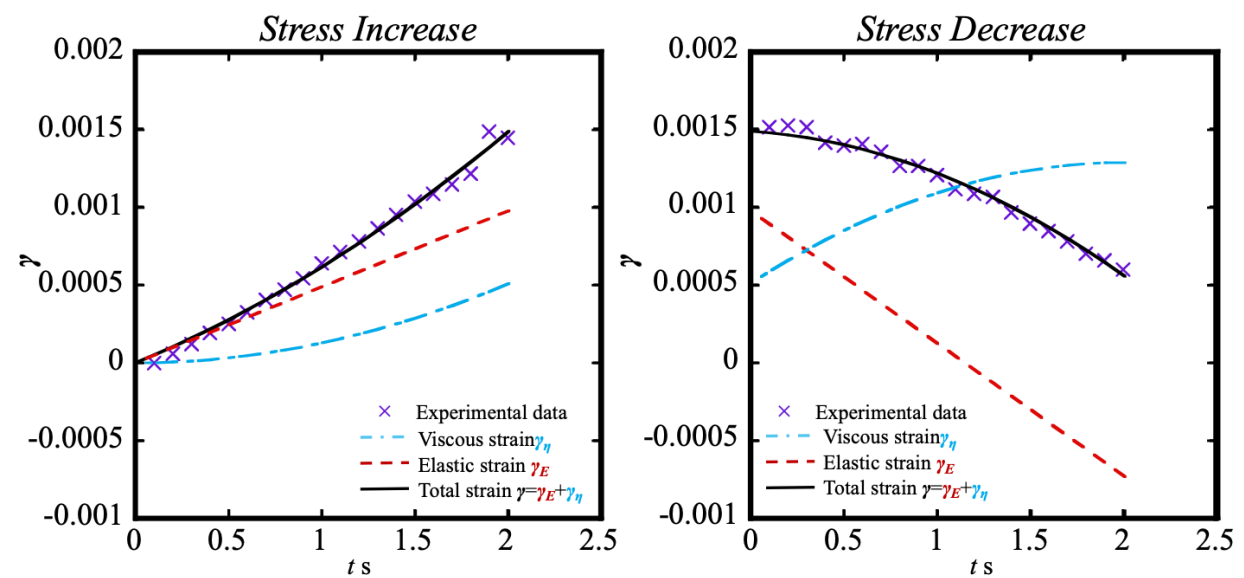

(d) $a=0.5 \mathrm{~Pa} / \mathrm{s}$

Figure 7: ...continued

the evolution of the strain in the stress decreasing segment, where the time corresponding to the maximum applied stress $\left(\tau_{\max }\right)$ is zero (i.e., $\left.t=0\right)$. When the stress-ramp rate is small, the strain first increases and then decreases in the stress decreasing segment. Moreover, the maximum value of the strain is attained for the smallest stress-ramp rate. This result indicates that the strain in the decreasing segment is influenced by the history of the viscous-dominated regime in the increasing segment. We observe that the single relaxation Maxwell model fits the experimental data well in case of the stress decreasing segment also. However, the difference between the experimental data and calculated strain is much more in the stress decreasing segment as opposed to that in the increasing segment, particularly for small stress-ramp rates. These results indicate that the stress decreasing segment is influenced by the flow history in the increasing segment, and the response changes the non-linear behavior by working the complex mechanism of the recovery of the structure.
Figure 8 shows the influence of the stress-ramp rate on the viscosity and elasticity obtained from the single relaxation Maxwell model at a maximum applied stress of $1 \mathrm{~Pa}$. Regardless of the surface roughness, the viscosity decreases as the stress-ramp rate increases for both the segments of increasing and decreasing stress. There is a shear-rate dependence for viscosity due to the accelerated shear flow of the sample resulting from the increase in the stress-ramp rate and not because of external factors such as wall-slip, since the rough surface shows the same result as the smooth surface. A large viscosity at small stress-ramp rates could also be owing to the jamming phenomena that induces interactions between the emulsified particles giving rise to shear. In the next section, we discuss whether the shear rate dependence or the time dependence of viscosity is more dominant below the yield point. For the smooth plate, the elasticity in both the segments of increasing and decreasing stress increases with the stress-ramp rate. However, for the rough plate, while the elasticity $E_{\text {up }}$ in the 


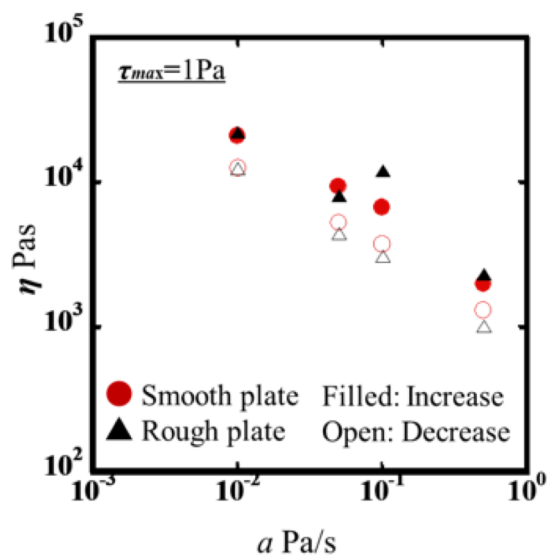

(a) Viscosity

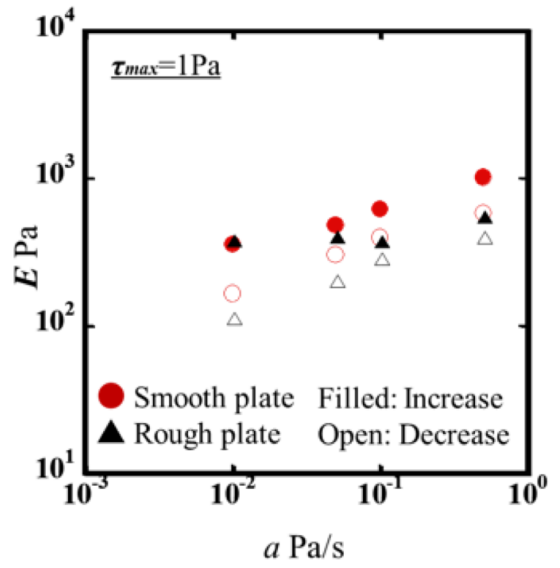

(b) Elasticity

Figure 8: Shear-rate and stress dependences of viscosity and elasticity from single relaxation Maxwell model fitting (for $\tau_{\max }=1 \mathrm{~Pa}$ ). (a) Viscosity and (b) elasticity, for both the segments of increasing and decreasing stress. The viscosity and elasticity in the decreasing stress segment are lower than those in the increasing stress segment because of the flow history in the increasing segment

increasing stress segment is constant, the elasticity $E_{\text {down }}$ in the decreasing stress segment increases with the stressramp rate. The increase in elasticity for the smooth plate is probably because of the following reason: the time for applying external stress shortens as the stress-ramp rate increases, and hence, it is possible that the gel network is stored without destruction in the slip layer. The constant $E_{u p}$ in the increasing stress segment for the rough plate is consistent with the results reported by N'Gouamba et al. [33], who found that the elastic modulus in the solid regime is constant. The elasticity $E_{\text {down }}$ in the decreasing stress segment shows the same behavior irrespective of the surface roughness, which implies that it is an intrinsic property of the sample. Note that both the viscosity $\eta_{\text {down }}$ and elasticity $E_{\text {down }}$ in the decreasing stress segment decrease because of the flow history in the increasing segment. Fur-

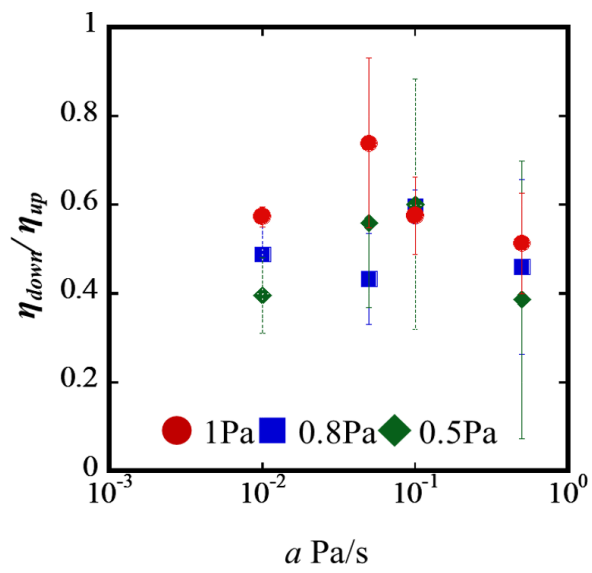

(a) Viscosity ratio

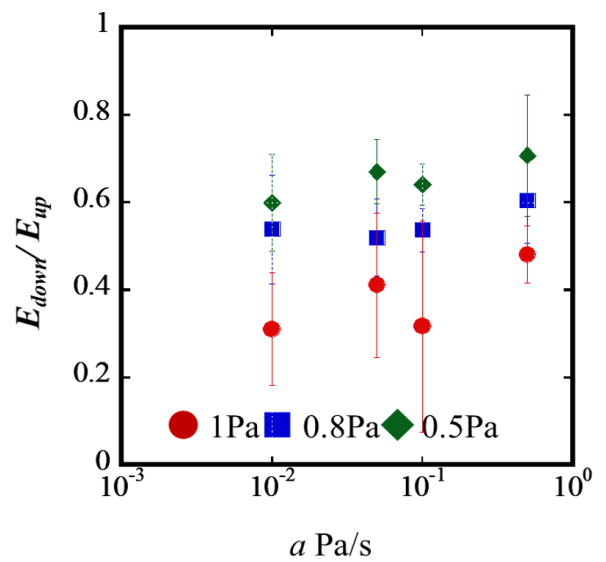

(b) Elasticity ratio

Figure 9: Evaluation of the flow history (time dependence and shearrate dependence) as a function of the stress-ramp rate for different maximum applied stress. (a) The viscosity ratio and (b) the elasticity ratio (between the coefficients in the segments of increasing and decreasing stress)

thermore, both the viscosity $\eta_{u p}$ in the increasing stress segment and the viscosity $\eta_{\text {down }}$ in the decreasing stress segment have the same slope. The elasticity $E_{\text {down }}$ in the decreasing stress segment is, however, smaller than the elasticity $E_{u p}$ in the increasing stress segment. This is probably owing to the destruction of the structure induced by the long term history of the flow in the increasing stress segment, which in turn lowers the elasticity of the sample.

To quantitatively evaluate the influence of the flow history, we calculated the viscosity ratio $\eta_{\text {down }} / \eta_{u p}$ and elasticity ratio $E_{\text {down }} / E_{\text {up }}$, as the flow history is essentially the change in these ratios. Figure 9 shows the stress-ramp rate dependence of the viscosity and elasticity ratios for three values of the maximum applied stress. From Figure 9(a), at any maximum applied stress, the viscosity ratio $\eta_{\text {down }} / \eta_{\text {up }}$ is less than 1 due to the fact that the viscosity in the decreasing segment drops more than in the increasing segment. 
In the condition of $0.5 \mathrm{~Pa}$ and $0.001 \mathrm{~Pa} / \mathrm{s}$, the effect of flow history in the diminishing of the viscosity reaches up to a $40 \%$ declination. From $0.05 \mathrm{~Pa} / \mathrm{s}$ to $0.5 \mathrm{~Pa} / \mathrm{s}$, the viscosity ratio shows a decreasing trend when increasing the stressramp rate. This is thought to occurs due to a decrease in viscosity caused by accelerated shear, as in shear thinning. In the elasticity ratio $E_{\text {down }} / E_{\text {up }}$ shown in Figure $9(\mathrm{~b})$, the elasticity ratio is elevated at high stress-ramp rate for all maximum applied stresses. Regarding the elastic modulus ratio, the maximum applied stress of 1 Pa shows an outrageous flow history. This suggests that the microstructure of the sample may be destroyed when a large shear stress is applied for a long time.

Therefore, we noted that the shear-rate dependence of the viscoelastic properties depends on the maximum applied stress and the stress-ramp rate. In the next section, we investigate the influence of the time dependence on the viscoelastic characteristics below the yield point.

\subsection{Time dependence of viscosity and elasticity from single relaxation Maxwell model}

The time dependence of the viscoelastic properties of the sample is investigated in this section. We introduced a creep test between the segments of increasing and decreasing stress in the hysteresis loop test. Figure 10 shows the viscosity and elasticity ratios before and after the creep test. The stress-ramp rate and maximum applied stress was fixed at $0.1 \mathrm{~Pa} / \mathrm{s}$ and $1 \mathrm{~Pa}$, respectively. In Figure 10, the flow history of the shear flow in the stress increasing segment is relaxed over time and the viscosity ratio reaches close to 1 . The decrease in the viscosity ratio can be explained by the destruction of the gel structure over the creep time. We observed that the decrease in the elasticity ratio from 1 to $1200 \mathrm{~s}$ of creep time is quite small. We believe this is because in this case the destruction of the structure with time is less than that under other conditions inducing accelerated shear flows. However, the time dependance of the accelerated shear flow may influence the destruction of the sample structure because the elasticity ratio is already small at a maximum applied stress of $1 \mathrm{~Pa}$ and stress-ramp rate of $0.01 \mathrm{~Pa} / \mathrm{s}$.

Therefore, we can conclude that the viscosity below the yield point in the $\alpha$-gel $\mathrm{O} / \mathrm{W}$ emulsion is influenced by the shear-rate dependence of the accelerated shear flow (which is related to the stress-ramp rate) rather than the time dependence. Additionally, it is clarified that the elasticity is influenced by the destruction of the gel-network.

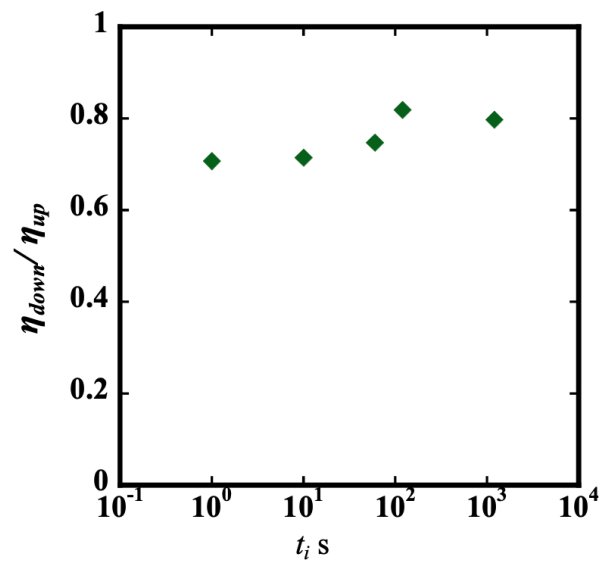

(a) Viscosity ratio

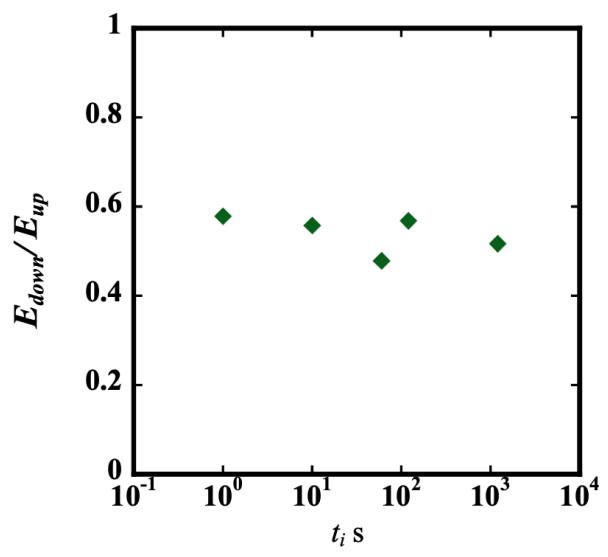

(b) Elasticity ratio

Figure 10: Influence of the creep time on the flow characteristics below the yield point for a maximum applied stress of $1 \mathrm{~Pa}$ and stress-ramp rate of $0.1 \mathrm{~Pa} / \mathrm{s}$. (a) The viscosity ratio and (b) the elasticity ratio. The creep time hardly influences the flow history of the viscoelastic characteristics

\subsection{Influence of viscoelastic behavior below yield point on yield characteristics}

We aim to clarify the influence of the viscoelastic behavior below the yield point on the yield characteristics. The stressramp test, in which the stress increases at a constant rate, was conducted to induce the slip and true yield stresses (see Figure 11). To remove the edge effect and reveal solely the stress associated with the wall-slip, Zhang et al. [28] showed $\boldsymbol{\tau}-\boldsymbol{\tau}_{\boldsymbol{y}}$ vs shear rate for the different diameters and various samples by conducting the series of creep tests. The slope in the slip regime was 1 in all conditions. Although we arranged $\boldsymbol{\tau}-\boldsymbol{\tau}_{y s}$ vs shear rate like their results, the slope in the slip regime was not 1 and it changed with increasing stress. It was considered that our tests have different flow fields in the slip regime than the creep test of Zhang et al. If we understand the difference in detail, the slip velocity 


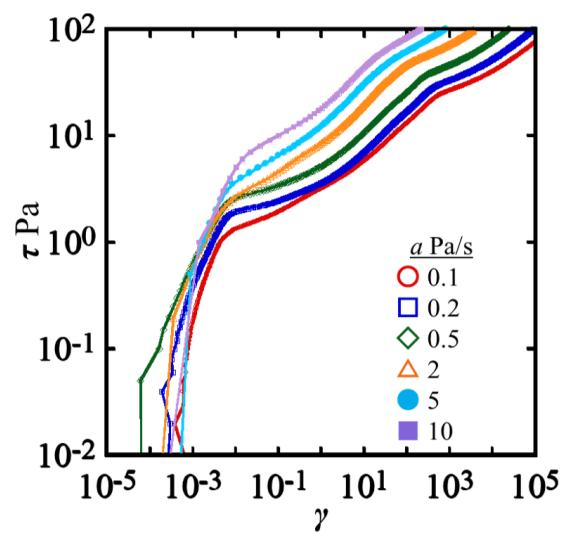

(a) Slip yield stress

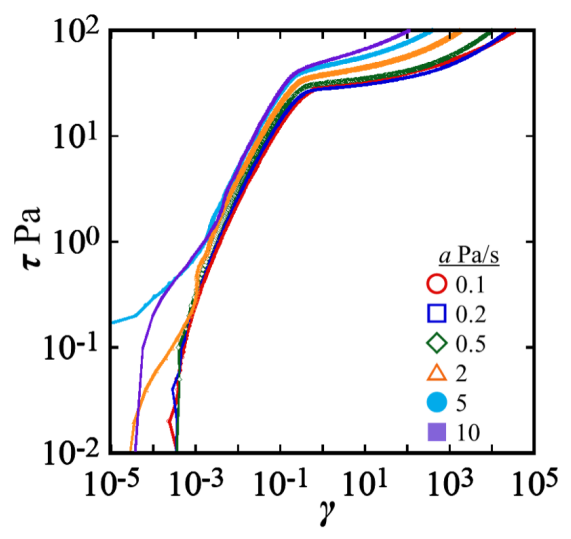

(b) True yield stress

Figure 11: Stress-strain curves from the stress-ramp test. (a) Slip yield stress and (b) true yield stress

and the origin of the viscosity would be revealed. Figure 12 shows the slip/true yield stress and the slip/true yield strain obtained from Figure 11 as a function of the stress-ramp rate. Figure 12(a) shows the results for the smooth surface, which exhibits slip behavior. We observe that the slip yield stress increases as the stress-ramp rate increases, while the slip yield strain is constant below $10 \mathrm{~Pa} / \mathrm{s}$. We found that the slip yield occurred during the critical strain and not stress for the $\alpha$-gel O/W emulsion. Figure 12(b) shows the results for the rough surface, which exhibits true yield behavior. We observe that the true yield stress increases slightly while the true yield strain decreases as the stressramp rate increases. Thus, there is no critical stress or strain that occurs during the true yield for the $\alpha$-gel $\mathrm{O} / \mathrm{W}$ emulsion. It is predicted that the true yield involves more complex behavior than the slip yield.

Before clarifying the viscoelastic behavior at the onset of yield, the limits of the applicability of the Maxwell model below the yield stress are examined. In the stress-strain curves obtained from the stress-ramp test, the range from

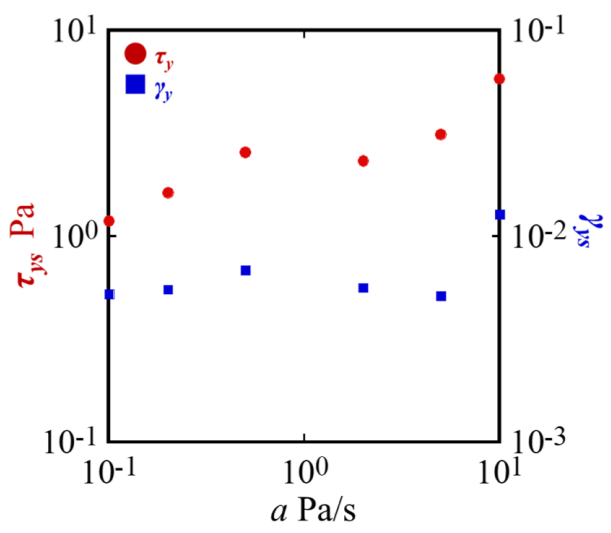

(a) Slip yield stress/strain

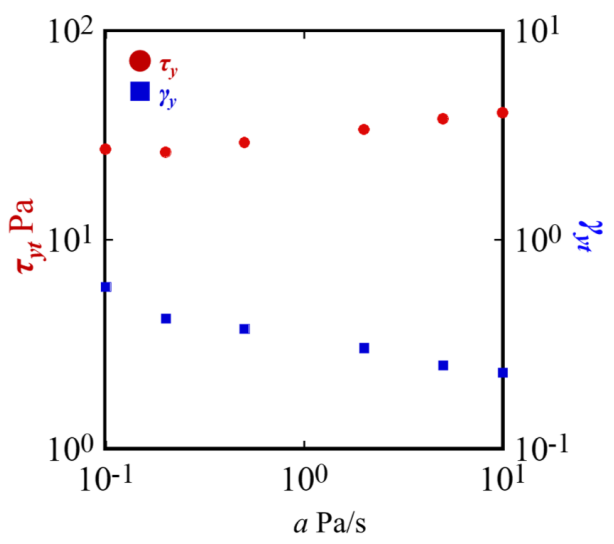

(b) True yield stress/strain

Figure 12: Influence of stress-ramp rate on (a) slip yield stress/strain and (b) true yield stress/strain

the start of the experiment to a certain stress $\tau_{\max }$ was used to be fitted by the Maxwell model. The high end stress of this fitting range $\tau_{\max }$ was selected from $0.5 \mathrm{~Pa}$ to the yield stress and the Maxwell model fitting was applied for each stress range. The correlation coefficient $\mathrm{R}^{2}$ between the Maxwell model and experimental data was calculated to examine the accuracy of the Maxwell model approximation. Figure 13 shows the correlation coefficient as a function of the maximum applied stress in the fitting range $\tau_{\max }$. The corresponding stress-strain curves are also shown in the figure. The horizontal axis $\boldsymbol{\tau}_{\max }$ means that the range of fitting is extended from zero to $\boldsymbol{\tau}_{\max }$, which is related to the correlation coefficient $R^{2}$ on the first vertical axis. Similarly, The horizontal axis $\boldsymbol{\tau}$ refers to the stress in the stress-ramp test and is related to the strain $\gamma$ in the second vertical axis. In other words, in Figure 13(a), the value of $\mathrm{R}^{2}$ at $\tau_{\max }=0.3$ Pa shows the result of the Maxwell model fitting for the range from zero to $0.3 \mathrm{~Pa}$. In Figure 13(a), the correlation coefficient is approximately 1 for a maximum applied stress between $0.5 \mathrm{~Pa}$ and $1.9 \mathrm{~Pa}$, which implies that the Maxwell model can fit the strain behavior before 


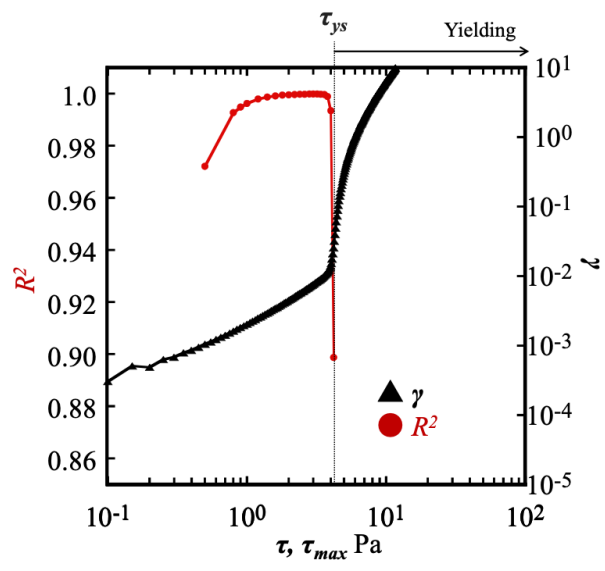

(a) Slip yield stress

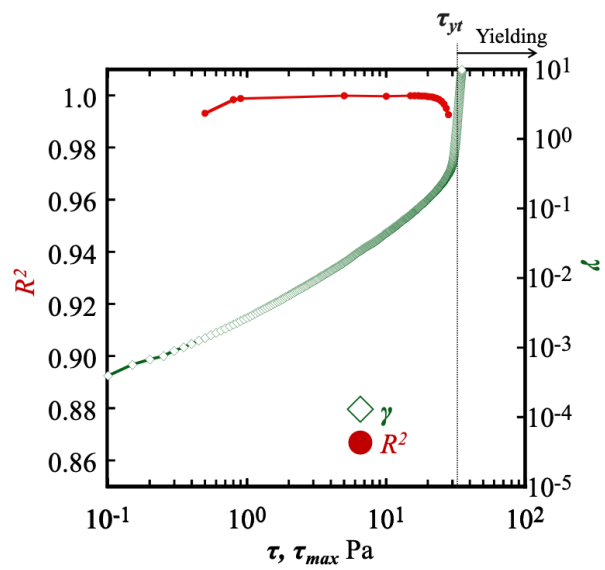

(b) True yield stress

Figure 13: Applicable range of the single relaxation Maxwell model. The correlation coefficient $R^{2}$ decreases just before the yield point. (a) Slip yield stress and (b) true yield stress. In both cases, the strain behavior can be approximated with high accuracy by the Maxwell model in most shear stress ranges below the yield stress

the slip yield stress. However, the correlation coefficient decreases to 0.90 just before $4.0 \mathrm{~Pa}$ of the slip yield stress. The decreasing tendency of correlation coefficient is considered to occurs due to the mechanical response in the sample changes from linear to nonlinear by the slip yield. That is why the Maxwell model cannot demonstrate the strain behavior over the slip yield stress. In Figure 13(b), the correlation coefficient before the true yield is about 1.0, which means that the Maxwell model, i.e. viscoelastic fluid model, can fit the strain behavior well even in the solid regime, although the regime is recognized as the elastoplastic body [33]. The correlation coefficient starts to decrease just before the true yield, and the fitting cannot be applied for a stress larger than the true yield. In order to clarify the effect of viscoelasticity on yielding, we used the fitting results before the correlation coefficient started to decrease.

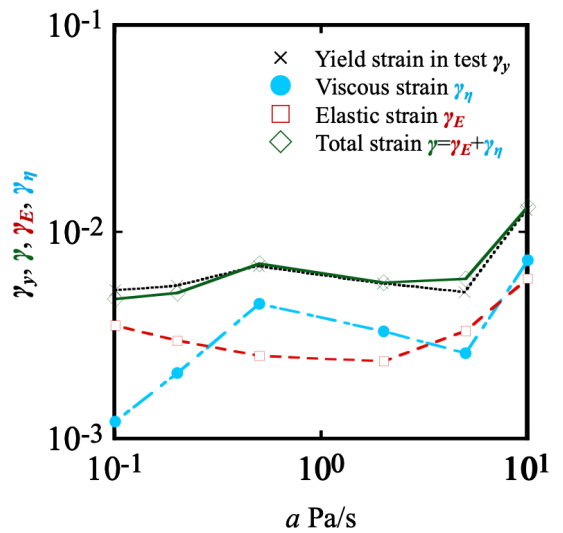

(a) Viscoelastic contribution in slip yield strain

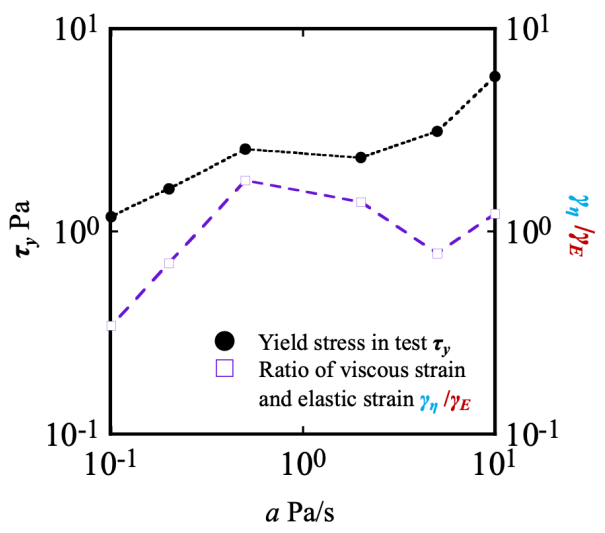

(b) Relationship between slip yield stress and ratio of viscous and elastic strains

Figure 14: Strain contribution of viscosity and elasticity to the slip yield characteristic in the stress-ramp test. (a) The viscoelastic contribution in the slip yield strain and (b) the relationship between the slip yield stress and the ratio of viscous and elastic strains. The deviation of the experimental yield strain from the Maxwell model is linked to the viscous strain. The yield stress for each stress-ramp rate is also influenced by the viscous strain

The strain required to determine the slip and true yield points was obtained by fitting the results of the stress-ramp test with the Maxwell model. Figure 14(a) shows the slip yield strain as a function of the stress-ramp rate calculated using the viscosity and elasticity obtained from the fitting. The qualitative trends of the viscous strain and total strain, which is the sum of the viscous and elastic strains (i.e., the yield strain), are in good agreement. To clarify the relationship between the yield stress and the viscoelastic contribution at the time of yield occurrence, Figure 14(b) shows the yield stress as well as the ratio of the viscous strain to elastic strain as functions of the stress-ramp rate. The increase in the strain ratio as well as the yield stress during yielding are linked to each other. Therefore, the shear-rate dependence governs the flow history below the yield point 


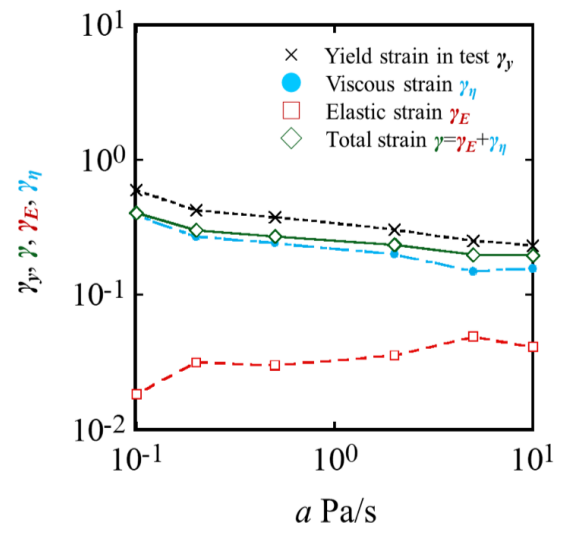

(a) Viscoelastic contribution in true yield strain

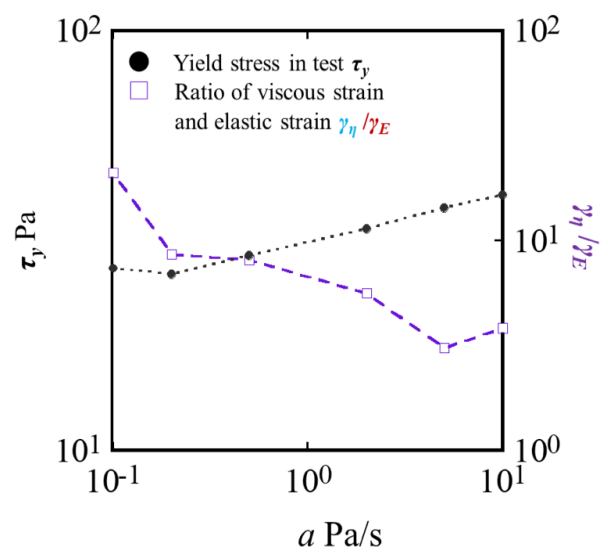

(b) Relationship between true yield stress and ratio of viscous and elastic strains

Figure 15: Strain contribution of viscosity and elasticity to the true yield characteristic in the stress-ramp test. (a) The viscoelastic contribution in the true yield strain and (b) the relationship between the true yield stress and the ratio of the viscous and elastic strains

for the $\alpha$-gel O/W emulsion. Consequently, the yield stress is not constant, whereas the yield strain varies with the stress-ramp rate because the viscous flow influences the strain significantly. Figure 15 shows the contribution of the viscous and elastic strains to the true yield strain. In Figure 15(a), as the stress-ramp rate increases, the elastic strain increases slightly, whereas the viscous strain decreases. The theoretical total strain shows the same qualitative trend as the experimental true yield strain but quantitatively they are different. As mentioned in the Introduction, this regime exhibits complex behavior, including plastic strain and linear-nonlinear transitions. Note that these influences were not evaluated by the single relaxation Maxwell model. Figure 15(b) is the same as Figure 14(b) except it is for the true yield case. Although the slip yield stress increases by contributing to the viscous strain, the true yield stress increases by contributing to the elastic strain. It is difficult to describe the exact behavior of true yield stress from these results. For evaluating the true yield, a more detailed analysis method is required. Considering Figure 13, the Maxwell model fitting could be applied to the strain not only before the slip yield but also to the true yield. If the Maxwell model could be applied to the partially divided strain in the solid regime, it will allow a more quantitative evaluation of the linear-nonlinear as well as solid-fluid transitions, and plastic strain with increasing the stress.

\section{Conclusions}

In this study, we conducted a hysteresis loop test and a stress-ramp test to investigate the influence of the flow characteristics below the slip yield point, and the relationship between the flow characteristics and the slip yield. Using the single relaxation Maxwell model to fit the strain behavior below the slip/true yield point, the shear-rate dependence, stress dependence, and time dependence of the flow characteristics were evaluated quantitatively. Our findings are summarized below.

In the hysteresis loop test for the smooth plate, the viscosity in both the segments of increasing and decreasing stress decreases with the stress-ramp rate. The change in viscosity is the same for both the smooth and rough plates, which implies that in this case the original characteristics of the sample were evaluated. However, the elasticity in the stress increasing segment did not increase with the stress-ramp rate for the rough plate. Since the flow history is below the slip yield stress, the viscosity in the decreasing stress segment is close to that in the increasing stress segment at a high stress-ramp rate. The elasticity in the decreasing stress segment decreases more than the elasticity in the increasing stress segment as the stress-ramp rate increases, except for the maximum applied stress of 1 $\mathrm{Pa}$. Thus, we found that a small applied stress and a high stress-ramp rate cannot explain the flow history in terms of the viscous flow of the sample for the smooth surface. In addition, the time dependence hardly influences the flow history in case of the slip yield behavior. To investigate the influence of the flow history below the slip yield point on the yield characteristics, a stress-ramp test and Maxwell model fitting were conducted. In the stress-ramp test, the slip yield stress increases as the stress-ramp rate increases, while the slip yield strain remains constant. This increase in the slip yield stress is related to the contribution of the viscous strain to the total accumulated strain, which indicates that the shear-thinning below the slip yield point controls the perturbation of the slip yield stress. On the 
other hand, the stress-ramp rate dependence of the true yield is difficult to explain only in terms of the contribution of the viscoelastic characteristics. However, the strain behavior is fitted well by the Maxwell model, which in turn implies that the yield phenomenon could be more easily understood by replacing the conventional plastic strain with viscous strain.

Conflict of interest: Authors state no conflict of interest.

\section{References}

[1] Bingham EC. Fluidity and plasticity. Mcgraw-Hill Book Company, Inc.. NY;1922.

[2] Coussot P. Bingham's heritage. Rheol Acta. 2017;56:163-176.

[3] Fourmentin M, Ovarlez G, Faure P, Peter U, Lesueur D, Daviller D, Coussot P. Rheology of lime paste-a comparison with cement paste. Rheol Acta. 2015;54:647-656.

[4] Weston JS, Harwell JH, Grady BP. Rheological characterization of yield stress gels formed via electrostatic heteroaggregation of metal oxide nanoparticles. Soft Matter. 2017;13:6743-6755.

[5] Masalova, I, Malkin AY, Foudazi R. Yield stress of emulsions and suspensions as measured in steady shearing and in oscillations. Appl Rheol. 2008;18:44790-44798.

[6] Bonn D, Denn MM, Berthier L, Divoux T, Manneville S. Yield stress materials in soft condensed matter. Rev Mod Phys. 2017;89:035005-035045.

[7] Varges PR, Costa MC, Fonseca BS, Naccache MF, Mendes SRP. Rheological Characterization of Carbopol Dispersions in Water and in Water/Glycerol Solutions. Fluids. 2019;4:3-23.

[8] Appel EA, Tibbitt MW, Webber MJ, Mattix BA, Veiseh O, Langer R. Self-assembled hydrogels utilizing polymer-nanoparticle interactions. Nat Commun. 2015;6:6295-6304.

[9] Nikko Chemicals Co. Ltd., Nippon Surfactant Indusiries Co Ltd., Toshiki Pigment Co., Ltd. Keshouhin Handbook. Chuo Printing Co., Ltd. Niigata;1996 (in Japanese).

[10] Møller PCF, Mewis J, Bon Daniel. Yield stress and thixotropy: on the difficulty of measuring yield stresses in practice. Soft Matter. 2006;2:274-283.

[11] Nelson AZ, Ewoldt RH. Design of yield-stress fluids: a rheologyto-structure inverse problem. Soft Matter. 2017;13:7578-7594.

[12] Kalyon DM, Yaras P, Aral B, Yilmazer U. Rheological behavior of a concentrated suspension: A solid rocket fuel simulant. J Rheol. 1993;37:35-53.

[13] Compton BG, Lewis JA. 3D-printing of lightweight cellular composites. Adv Mater. 2014;26:5930-5935.

[14] Giuseppe ED, Corbi F, Funiciello F, Massmeyer A, Santimano TN, Rosenau M, Davaille A. Characterization of Carbopol ${ }^{\circledR}$ hydrogel rheology for experimental tectonics and geodynamics. Tectonophysics. 2015;642:29-45.

[15] Dinkgreve M, Paredes J, Denn MM, Bonn D. On different ways of measuring "the" yield stress. J Non-Newtonian Fluid Mech. 2016;238:233-241.

[16] Dinkgreve M, Fazilati M, Denn MM, Bonn D. Carbopol: From a simple to a thixotropic yield stress fluid. J Rheol. 2018;62:773780.
[17] Moller P, Fall A, Chikkadi V, Derks D, Daniel B. An attempt to categorize yield stress fluid behaviour. Phil Trans R Soc. 2009;A 367:5139-5155.

[18] Coussot P, Nguyen QD, Huynh HT, Daniel B. Viscosity bifurcation in thixotropic, yielding fluids. J Rheol. 2002;46:573-589.

[19] Ovarlez G, Tocquer L, Bertrand F, Coussot P. Rheopexy and tunable yield stress of carbon black suspensions. Soft Matter. 2013;9:5540-5549.

[20] Grenard V, Divoux T, Taberlet N, Manneville S. Timescales in creep and yielding of attractive gels. Soft Matter. 2014;10:1555-1571.

[21] Larson GR, Wei Y. A review of thixotropy and its rheological modeling. J Rheol. 2019;63:477-501.

[22] Moghimi E, Petekidis G. Mechanisms of two-step yielding in attractive colloidal glasses. J Rheol. 2020;64:1209-1225.

[23] Cloitre, M, Bonnecaze RT. A review on wall slip in high solid dispersions. Rheol Acta. 2017;56:283-305.

[24] Meeker SP, Bonnecaze RT, Cloitre M. Slip and flow in soft particle pastes. Phys Rev Lett. 2004;92:198302-198305.

[25] Meeker SP, Bonnecaze RT, Cloitre M. Slip and flow in pastes of soft particles: Direct observation and rheology. J Rheol. 2004;48:1295-1320.

[26] Ballesta P, Besseling R, Isa L, Petekidis G, Poon WCK. Slip and flow of hard-sphere colloidal glasses. Phys Rev Lett. 2008;101:258301-258304.

[27] Ballesta P, Besseling R, Isa L, Petekidis G, Poon WCK. Wall slip and flow of concentrated hard-sphere colloidal suspensions. J Rheol. 2012;56:1005-1037.

[28] Zhang X, Lorenceau E, Bourouina T, Basset P, Oerther T, Ferrari $M$, Rouyer F, Goyon J, Coussot P. Wall slip mechanisms in direct and inverse emulsions. J Rheol. 2018;62:1495-1513.

[29] Coussot P, Tabuteau H, Chateau X, Tocquer L, Ovarlez G. Aging and solid or liquid behavior in pastes. J Rheol. 2006;50:975-994.

[30] Tabuteau H, Baudez JC, Chateau X, Coussot P. Flow of a yield stress fluid over a rotating surface. Rheol Acta. 2007;46:341355.

[31] Saramito P. A new elastoviscoplastic model based on the Herschel-Bulkley viscoplastic model. J Non-Newtonian Fluid Mech. 2009;158:154-161.

[32] Dimitriou C), Ewoldt RH, McKinley GH. Describing and prescribing the constitutive response of yield stress fluids using large amplitude oscillatory shear stress (LAOStress). J Rheol. 2013;57:2770.

[33] N'Gouamba E, Goyon J, Coussot P. Elastoplastic behavior of yield stress fluids. Phys Rev Lett. 2019;4:123301-123318.

[34] Watanabe K, Inoue H, Teshigawara T, Kimura T. $\alpha$-Gel Prepared in Sodium Methyl Stearoyl Taurate/Behenyl Alcohol/Water SystemCharacterization of Structural Changes with Water Concentration. J Oleo Science 2012;61:29-34.

[35] Nabata Y, “Keshohin no Reoroji”, Yoneda Publishing, Tokyo;2015 (in Japanese).

[36] Homma I, Sato Y, Takahashi T, Noda K, Sogabe A. A Study on Yield Behavior and Stress Relaxation of $\alpha$-Gels. Nihon Reoroji Gakkaishi (J Soc Rheol Jpn). 2018:46;67-75 (in Japanese).

[37] Mewis J. Thixotropy - A general review. J Non-newton Fluid Mech. 1979;6:1-20.

[38] Rubens RF, Diogo EVA, Admilson TF, Cezar ORN. The yielding and the linear-to-nonlinear viscoelastic transition of an elastoviscoplastic material. J Rheol. 2017;61:893-903. 